\title{
Feline Pansteatitis: A Report of Five Cases
}

Pansteatitis (yellow fat disease, panniculitis, steatitis) is an inflammatory disease of adipose tissue throughout the body (Holzworth 1987). It was first experimentally induced by Mason \& Dam in 1946 in cats fed a diet deficient in vitamin E and high in cod liver oil (Mason \& Dam 1946). It has since been reported as a clinical condition by several authors (Cordy \& Stillinger 1953, Watson et al. 1973, Gaskell et al. 1975, Summers et al. 1982, Hagiwara et al. 1986). Pansteatitis occurs naturally in cats, mink, and pigs as a result of vitamin E deficiency. Vitamin $\mathrm{E}$ ( $\alpha$-tocopherol) is a biological antioxidant found in vegetable oils (Holzworth 1987). It serves as a protector of the fats in the diet and in the body. Pansteatitis is caused by a mismatch between intake of unsaturated fatty acids and antioxidants, i.e. vitamin E. The ensuing peroxidation of the body fat causes a foreign body reaction with severe inflammation and cell death. The foremost clinical sign is hyperaesthesia or severe pain on palpation/handling, especially over the back and of the abdomen. The final diagnosis rests with the histological findings of the above-mentioned lesions in conjunction with acid-fast ceroid pigment (i.e. end-product of lipid peroxidation) in fat cells, in macrophages, in Langhans-type giant cells, and extracellularly (Holzworth 1987).

Treatment consists of a change of diet, vitamin E supplementation and occasionally corticosteroids. Prognosis is reported to be fair with treatment, but must be regarded as potentially poor depending on the severity of the disease. This paper describes the clinical findings in 5 histologically confirmed cases of pansteatitis in the cat. The purpose of this report is to alert the clinician to the possibility of finding cases with this disease among their feline patients.

Case 1. A 1 1 1/2-year-old domestic short-haired male cat was referred with a history of lethargy, fever, inappetence and tenderness over the dorsal muscles. The referring veterinarian had instituted a treatment of penicillin and corticosteroids 2 days previously.

On presentation the cat was depressed and had a rectal temperature of $39.3^{\circ} \mathrm{C}$. The abdomen and the dorsal muscles were extremely painful on palpation. The cat was sedated with a combination of xylazine and ketamine before radiographs of the abdomen and the lumbar vertebral column were taken. As no abnormalities were seen, the owner was recommended to continue treatment with antibiotics at home. The cat was returned 5 days later with a rectal temperature of $40.4^{\circ} \mathrm{C}$. Pain was still evident on palpation of the abdomen and over the dorsum.

Haematological examination revealed a total white blood cell count of 17,100 cells $/ \mu$ l with a differential count of 1,368 band neutrophils $/ \mu$, 13,851 segmented neutrophils/ $\mu 1,342$ eosinophils $/ \mu$ l and 1,539 lymphocytes $/ \mu$ l. Packed cell volume was $38 \%$ and haemoglobin $136 \mathrm{~g} / \mathrm{l}$. 
Blood chemistries showed blood urea nitrogen (BUN) $5.2 \mathrm{mmol} / 1$, blood glucose $10.7 \mathrm{mmol} / 1$ and alanine aminotransferase (ALAT) 0.6 $\mu \mathrm{kat} / \mathrm{l}$.

The cat was given antibiotics and intravenous fluids for 2 days, but no improvement occurred and euthanasia was carried out.

Histological evaluation of a subcutaneous fat biopsy demonstrated widespread necrotic areas and severe interstitial inflammation with accumulation of mononuclear cells and macrophages containing acid-fast pigment (ceroid). Perivascular cuffs of neutrophils were also seen.

It was later discovered that the cat's diet had consisted of fish, oatmeal and canned carrots and peas since an early age.

Case 2. A 6-year-old domestic short-haired male cat was presented with a history of intensive vomiting for $6 \mathrm{~h}$.

The cat was obese but was otherwise in a good condition. Abdominal palpation revealed no abnormalities. Radiographic examination of the abdomen showed an abnormal intestinal gas distribution. A barium study of the gastrointestinal canal was suggestive of a string foreign body. The following day a laparotomy was performed and a sewing thread was found, extending from under the tongue to the colon. In addition, the entire visible adipose tissue had a distinct yellow discolouration. A preliminary diagnosis of pansteatitis was made and the cat was euthanized at the owner's request. The cat had been eating solely fish for 6 years, but had converted to commercial cat food during the last year.

A biopsy of the subcutaneous fat was evaluated histologically and the following features were found: A diffuse inflammatory reaction with infiltration of neutrophils in the adipose tissue. Giant cells were also present, containing acidfast droplets of ceroid pigment. The pigment was observed in the interstitium between the fat cells as well.

Case 3. A one-year-old domestic short-haired male cat was presented with a one day history of inappetence, polydipsia and ataxia. The cat had reportedly resented handling for a couple of days. The cat's diet consisted exclusively of herring.

Physical examination revealed a moderately depressed cat with a rectal temperature of $40.2^{\circ} \mathrm{C}$. Auscultation of the heart and lungs was normal. Palpation of dorsal muscles elicited a painful response. Abdominal palpation revealed several small nodules throughout the cavity.

Haematological examination revealed a total white blood cell count of 68,500 cells $/ \mu$ l with a differential count of 7,535 band neutrophils $/ \mu$, 59,595 segmented neutrophils/ $\mu \mathrm{l}$, and 1,370 lymfocytes $/ \mu$ l. Packed cell volume was $35 \%$, haemoglobin $120 \mathrm{~g} / 1$, and erythrocyte count $6.1 \times 10^{12}$. Blood chemistry showed BUN 8.2 $\mathrm{mmol} / \mathrm{l}$, blood glucose $9.2 \mathrm{mmol} / \mathrm{l}$, alkaline phosphatase (ALP) $0.9 \mu \mathrm{kat} / \mathrm{l}$ and alanine aminotransferase (ALAT) below range.

The cat was treated with $5 \mathrm{mg}$ vitamin $\mathrm{E}$ im daily, intravenous fluids and ampicillin for 7 consecutive days. After this time, the skin began to peel off over the dorsum, and the cat's condition had deteriorated to the point where the owner chose euthanasia.

On necropsy a severe generalized pansteatitis with a histological appearance similar to case 1 , along with peripheral oedema and ascites was found. There were also erosions in the gastric mucosa.

Case 4. A $3 \frac{1}{2}$-year-old neutered female domestic short-haired cat was examined because of pain in the hindquarters. On palpation the cat showed severe pain over the abdomen, hindlegs, and hindquarters, especially over the lumbar region. Radiographs of the abdomen, the 
lumbar and the pelvic regions showed normal features. The cat's diet consisted solely of a variety of fish.

Two months later the cat was presented to the clinic because of an infected wound at the tail head. The cat was treated with tetracyclines and the diet was changed to Hill's Prescription Diet $\mathrm{c} / \mathrm{d}$. Vitamin E and selenium supplementation was also started. The cat was asymptomatic by the owner's judgment for the following $1 \frac{1}{2}$ years. It was then brought to the clinic because it resented handling. The cat was at that time severely obese. Radiographic examination of the lumbar area was repeated, and showed no abnormality. Two weeks later the skin over the back began to excoriate on palpation, and the cat was euthanized at the owner's request.

Necropsy revealed severe pansteatitis with typical histological appearance of the adipose tissue, especially subcutanously over the dorsum. Pleural and pericardial effusions and pulmonary atelectasis were also found.

Case 5. A one-year-old Persian female cat was presented because of inappetence, dyspnea and weight loss. The cat was emaciated and severely depressed. The hair coat had a dull appearance. On abdominal palpation several firm lumps were felt throughout the cavity. As the preliminary diagnosis was abdominal tumors (suspected alimentary leukosis) and the prognosis deemed poor, the cat was euthanized. It was later found out that the cat had been eating fish almost exclusively since the age of 10-12 weeks.

On post mortem examination severe pansteatitis with a pathognomonic histological picture of most of the subcutanous adipose tissue as well as the thoracic, abdominal and the perirenal fat was found. Ascites, pleural effusion, subcutaneous oedema, pulmonary atelectasis and centro-lobular hepatic necrosis were also found.
Pansteatitis is a nutritional disease caused by inadequate quantity of antioxidants, i.e. vitamin $\mathrm{E}$, in the presence of high levels of unsaturated fatty acids in the diet.

Most often affected cats have reportedly been fed mainly fish (Holzworth 1987), but occasional cases have been documented in which the diet contained a minimal quantity of fish (Watson et al. 1973, Summers et al. 1982). All the cases reported here had been fed a fishbased diet during a time period varying from 6 months to 6 years. One cat had exclusively been eating herring, and another mainly cod.

Pansteatitis is reportedly most often seen in young adult cats with a mean age of 2.5 years (Holzworth, 1987). This is in agreement with the findings in this study where the age ranged from 1 to 6 years. Females are reported to be more prone to pansteatitis than males (Holzworth 1987). The female/male ratio in this study was 2 to 3 .

The most striking feature on physical examination of the patients, as well as the owners' chief complaint on presentation, is the marked and severe pain response that handling and/or palpation elicits (Holzworth 1987). Pain seems to be most severe over the back, the tail head and in the abdomen. Three of the cats reported here were distinctly painful on abdominal and dorsal palpation. Case 5 was severely ill and therefore may not have reacted normally to painful stimuli. Case 2 was apparently asymptomatic when it ingested a string foreign body, which may indicate an earlier stage of the disease.

Inappetence, depression, fever and reluctance to move are other signs often observed by the owners of cats affected with pansteatitis (Watson et al. 1973, Gaskell et al. 1975, Holzworth 1987). These symptoms were seen in cases 1,3 and 5 of this study. Case 3 was polydipsic, a sign also reported by previous authors (Gaskell et al. 1975). Case 4 was severely obese, while case 5 was emaciated. Excoriation of the skin 
over the back was seen in 2 cases (Nos. 3 and 4). This finding has not been reported before to our knowledge.

Radiographic findings reported in one study included irregular radio densities of the subcutaneous, falciform and inguinal fat and a poor radiographic contrast within the abdomen (Gaskell, et al. 1975). Three of the 5 cases reported here underwent radiographic examination of the abdomen and the dorsal region. No abnormalities were found, except for the cat with the concomitant jejunal foreign body, where radiographs showed abnormal gas distribution within the intestines.

Post mortem examination was performed in 3 of the cases and subcutaneous biopsies were evaluated in the other 2 cases presented here. Macroscopical and histopathological findings were well in agreement with those described in previous studies (Gaskell et al. 1975, Holzworth 1987). The adipose tissue was yellow, firm and nodular. Histological examination showed necrosis of fat cells and severe inflammation of the interstitial tissue with neutrophils, giant cells and macrophages containing acid-fast droplets of ceroid pigment (Fig. 1). Ascites, found in 2 cases in this study (nos. 3 and 5), have been reported (Summers et al. 1982). Pleural effusion which was present in cases 4 and 5, and subcutaneous oedema in cases 3 and 5, have not been reported previously to our knowledge.

Treatment has reportedly consisted of exclusion of fish and/or liver from the diet, vitamin $\mathrm{E}$ supplementation and corticosteroids. Recommended doses of vitamin $\mathrm{E}$ varies between 10 and $25 \mathrm{IU} / \mathrm{kg}$ b.i.d. orally (Watson et al. 1973, Gaskell et al. 1975, Morgan 1988). As a preventive measure $80 \mathrm{IU}$ vitamin $\mathrm{E} / \mathrm{kg}$ diet has been recommended (Holzworth 1987). The use of corticosteroids to reduce the inflammatory reaction has been advocated by some, with doses ranging from $5-7.5 \mathrm{mg} / \mathrm{cat}$ to $2.5 \mathrm{mg} / \mathrm{kg}$ t.i.d. Handling of the cat should be minimized and subcutaneous injections avoided (Morgan 1988).

Prognosis varies with severity of the disease, but several reports claim successful outcome of the treatment in many cases (Gaskell et al. 1975, Summers et al. 1982). Treatment with vitamin E was attempted in cases 3 and 4 of this study. Case 3 was euthanized after 7 days of treatment with intravenous fluids and vitamin $\mathrm{E}$ injections. The cat deteriorated continuously, and the skin ruptured at several places. Cat no. 4 was successfully treated with tetracyclines, a change of diet, vitamin E and selenium supplementation, and remained, after initial remission, asymptomatic for 21 months before it relapsed. The cat was euthanized without a second therapeutic trial. Cases 1 and 2 were euthanized without adequate therapy. The poor clinical condition of case 5 did not permit therapy to be tried.

From this and other studies (Watson et al. 1973, Gaskell et al. 1975, Summers et al. 1982, Hagiwara et al. 1986) it seems safe to conclude that untreated, or too late diagnosed, feline pansteatitis is an ultimately fatal disease. A preliminary clinical diagnosis may be made in cats who display severe pain when touched, especially over the back and when the abdomen is palpated. Palpation of the abdomen and subcutaneous fat usually reveal firm nodules. A final diagnosis is made by finding typical histological lesions. Treatment consists of exclusion of fish and/or liver from the diet, vitamin E supplementation and often corticosteriods. Although not many recent reports appear in the literature, this is not a disease to be forgotten, and client education concerning feline nutrition cannot be overemphasized.

A. Tidholm, I. Karlsson, B. Wallius, Djursjukhuset Albano - Animal Hospital of Stockholm, Rinkebyvägen 23, S-182 36 Danderyd, Sweden. 


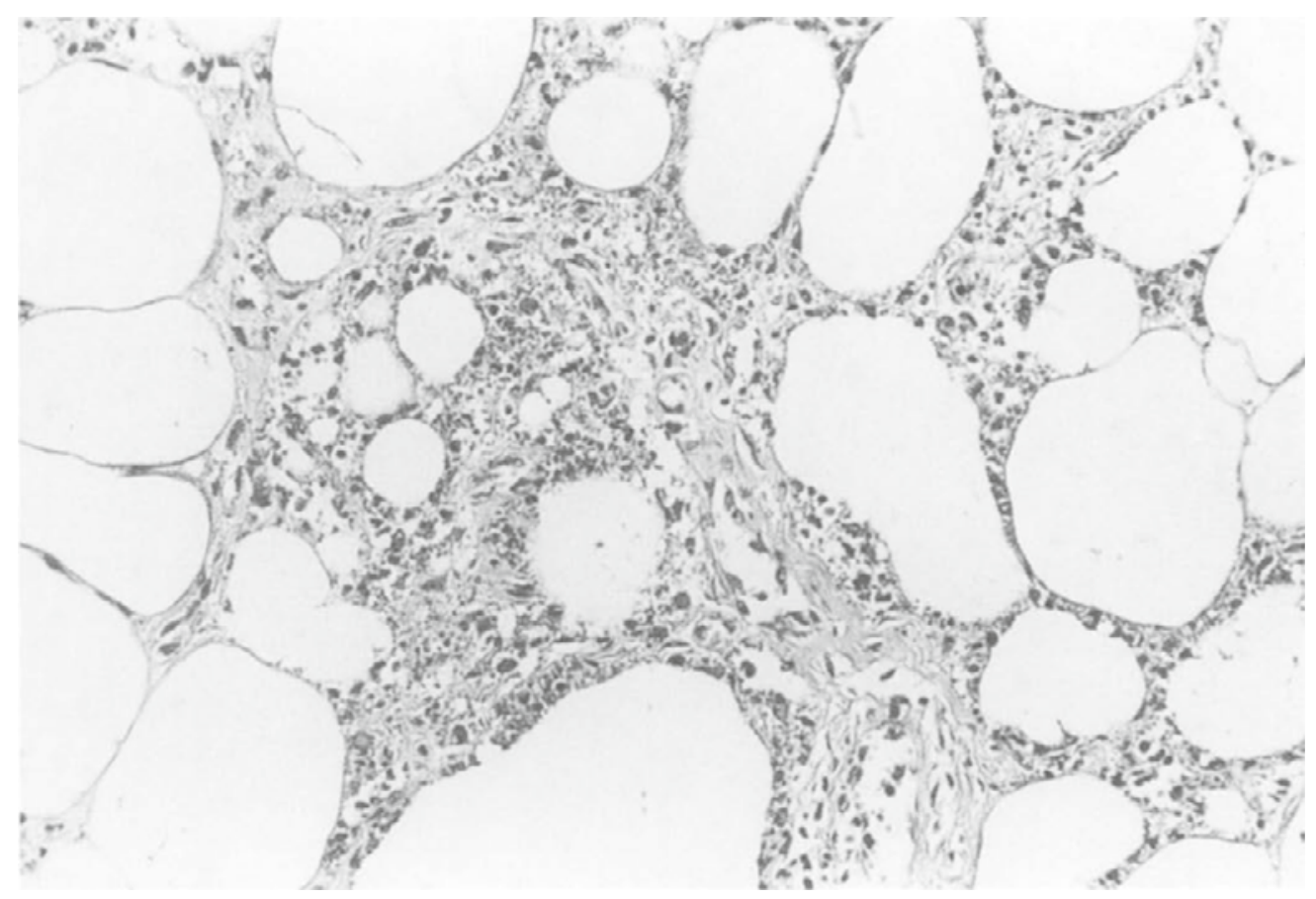

Figure 1. Histological section of subcutaneous steatitis with numerous deposits of acid-fast, ceroid pigment in the macrophages. Ziehl-Neelsen stain x 280.

\section{Acknowledgments}

Biopsy and postmortem examinations were performed at BioVet Diagnostic Laboratory. We would like to thank Dr. Lennart Jönsson (Uppsala) for preparing the micrograph.

\section{References}

Cordy DL, Stillinger CJ: Steatitis ("Yellow fat disease") in kittens. North. Amer. Vet. 1953, 34, 714-716.

Gaskell CJ, Leedale AH, Douglas SW: Pansteatitis in the cat: A report of four cases. J. Small Anim. Pract. 1975, 16, 117-121.

Hagiwara MK, Guerra JL, Maeoka MRM: Pansteatitis in a Cat. Feline Practice 1986, 16, 25-27.
Holzworth J: Diseases of the Cat: Medicine and Surgery. W. B. Saunders Company, Philadelphia, 1987.

Mason KE, Dam H: Histological changes in adipose tissue of cats fed a vitamin E deficient diet high in cod liver oil. Anat. Rec. 1946, 94, 265-288.

Morgan RV: Handbook of Small Animal Practice. Churchill Livingstone, New York, 1988.

Summers BA, Sykes G, Martin L: Pansteatitis mimicking infectious peritonitis in a cat. J. Amer. vet. Ass. 1982, 180, 546-549.

Watson ADJ, Porges WL, Huxtable CR, Ilkiw WJ: Pansteatitis in a cat. Austr. vet. J. 1973, 49, 388392

(Received September 7, 1995; accepted December 22, 1996).

Reprints may be obtained from: A. Tidholm, Djursjukhuset Albano, Rinkebyvägen 23, S-182 36 Danderyd, Sweden. 




